\title{
Surface Melting Curve Analysis with R
}

by Stefan Rödiger, Alexander Böhm and Ingolf Schimke

Abstract Nucleic acid Melting Curve Analysis is a powerful method to investigate the interaction of double stranded nucleic acids. Many researchers rely on closed source software which is not ubiquitously available, and gives only little control over the computation and data presentation. $\mathrm{R}$ in contrast, is open source, highly adaptable and provides numerous utilities for data import, sophisticated statistical analysis and presentation in publication quality. This article covers methods, implemented in the MBmca package, for DNA Melting Curve Analysis on microbead surfaces. Particularly, the use of the second derivative melting peaks is suggested as an additional parameter to characterize the melting behavior of DNA duplexes. Examples of microbead surface Melting Curve Analysis on fragments of human genes are presented.

\section{Introduction}

\section{Melting Curve Analysis}

Nucleic acid Melting Curve Analysis (MCA) is a central step in nucleic acid ${ }^{1}$ interaction studies, identification of specific DNA sequences after quantitative real-time PCR, genotyping or detection of Single Nucleotide Polymorphisms ${ }^{2}$ (SNP) both in solution and on surfaces (Ririe et al., 1997; Gundry et al., 2003; Sekar et al., 2005; Zhou et al., 2005; Rödiger et al., 2012b). A review of the literature revealed that there is an ongoing demand for new bioanalytical devices to perform MCAs. This includes lab-on-chip systems or the recently published VideoScan platform (Rödiger et al., 2012b, see following section). Some of these systems offer software solutions for MCA but often custom made software is required. Although bioanalytical devices break new ground to meet criteria like high multiplex levels or new detection-probe-systems the fundamental concept of MCA remains unchanged.

The MCA is a real-time monitoring of a heat-induced double stranded nucleic acid dissociation which can be monitored by the change of the referenced mean/median fluorescence intensity $(M F I)^{3}$ at a defined temperature (Figure 1).

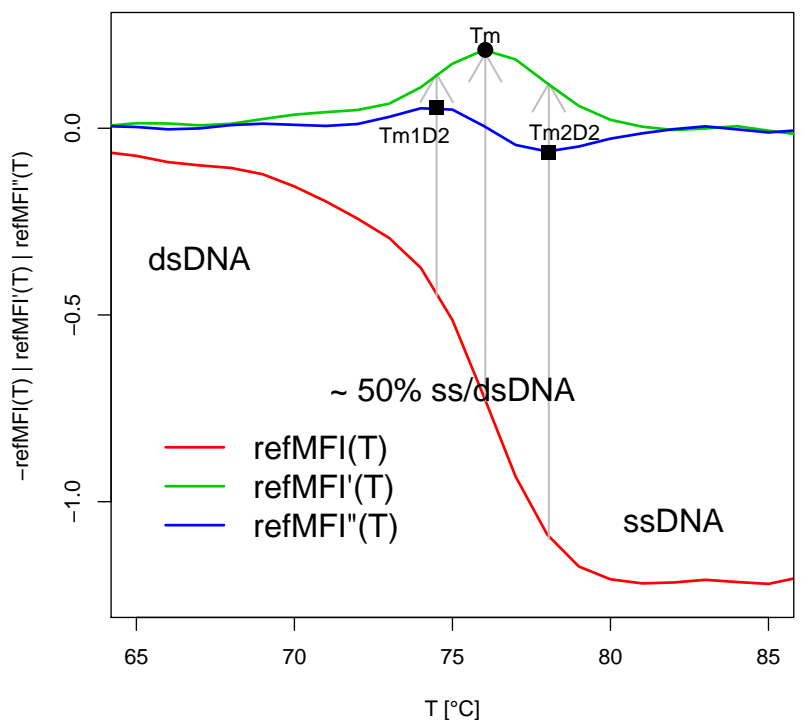

Figure 1: Melting curve and melting peaks of double stranded DNA. Raw data curves (refMFI(T) -), approximate first derivative $\left(\operatorname{refMFI} I^{\prime}(T)-\right)$ and second derivative (refMFI" $(T)-$ ). Peak values: $T m$ is the maximum of the first derivative. $T m_{1}^{D 2}$ and $T m_{2}^{D 2}$ are the extremes (a minimum and a maximum) of the second derivative. dsDNA, double stranded DNA; ssDNA, single stranded DNA.

\footnotetext{
${ }^{1}$ Nucleic acids herein refer to deoxyribonucleic acids (DNA) and ribonucleic acids (RNA).

${ }^{2}$ Single Nucleotide Polymorphisms (SNP) are caused by exchanges of single nucleotides.

${ }^{3}$ In the past absorbency was the quantitative measure. New devices measure fluorescence intensities mediated by nucleic acid-intercalating fluorophores (e.g., EvaGreen $\left.{ }^{\circledR}\right)$ or fluorophore labeled DNA probes (e.g., Molecular Beacons, Gašparič et al., 2010; Rödiger et al., 2012a). The values are often reported as RFU (Relative Fluorescence Units), MFI (Mean/Median Fluorescence Intensity) or refMFI (Referenced Mean/Median Fluorescence Intensity).
} 
By definition, the melting point $(\mathrm{Tm})$ is the inflection point of the melting curve. On molecular level circa $50 \%$ of the nucleic acids are dissociated at Tm. The melting peak (Equation 1) can be determined from the first negative derivative (Equation 2) of the melting curve. At this temperature peak the rate of change is maximal. The Tm is highly reproducible, thus can be used as a "characteristic identity" to distinguish nucleic acid species.

$$
\begin{aligned}
T m & =\max (\operatorname{refMFI}(T)) \\
\operatorname{refMFI}(T) & =-\frac{d(\operatorname{ref} M F I)}{d(T)}
\end{aligned}
$$

To the best of our knowledge we are the first to suggest the peak values, designated $T m_{1}^{D 2}$ and $T m_{2}^{D 2}$ (Equation 3 and 4), of the second derivative (Equation 5) as additional measures for the characterization of nucleic acid melting processes on microbead surfaces. They show the maximal rate of change of $\operatorname{refMFI^{\prime }}(T)$. The corresponding temperature values (abscissa) at the inflection points of ref $M F I^{\prime}(T)$ occur where ref $M F I^{\prime \prime}(T)$ reaches a (local) minimum and a (local) maximum, respectively (Figure 1). Both values offer additional quantitative measures to describe early and late phases of the melting process. The $T m_{1}^{D 2}$ quantifies the maximal acceleration and $T m_{2}^{D 2}$ the maximal deceleration of the dissociation process. The deceleration starts in the middle of the process, at $\mathrm{Tm}$.

$$
\begin{aligned}
& T m_{1}^{D 2}=\max \left(r e f M F I^{\prime \prime}(T)\right) \\
& \operatorname{Tm}_{2}^{D 2}=\min \left(r e f M F I^{\prime \prime}(T)\right) \\
& \operatorname{refMFI"}(T)=-\frac{d^{2}(\operatorname{ref} M F I)}{d(T)^{2}}
\end{aligned}
$$

\section{Surface Melting Curve Analysis}

In Rödiger et al. (2012b) we reported the VideoScan platform which provides a technology for various bioanalytical applications ${ }^{4}$ with the focus on highly multiplex kinetic analysis. The VideoScan platform consists of a fully automated fluorescence microscope, a modular software package for data acquisition and a heating/cooling-unit $(\mathrm{HCU})^{5}$ for micro volume $(\leq 20 \mu \mathrm{L})$ samples. We developed temperature controlled assays to detect and analyze nucleic acid probes on the surface of microbeads.

In brief, different thermo-tolerant microbead populations, defined by varying ratios of two impregnated fluorophores, are included in the reaction. Each microbead population presents gene specific capture probes on their surfaces (Figure 2).

A

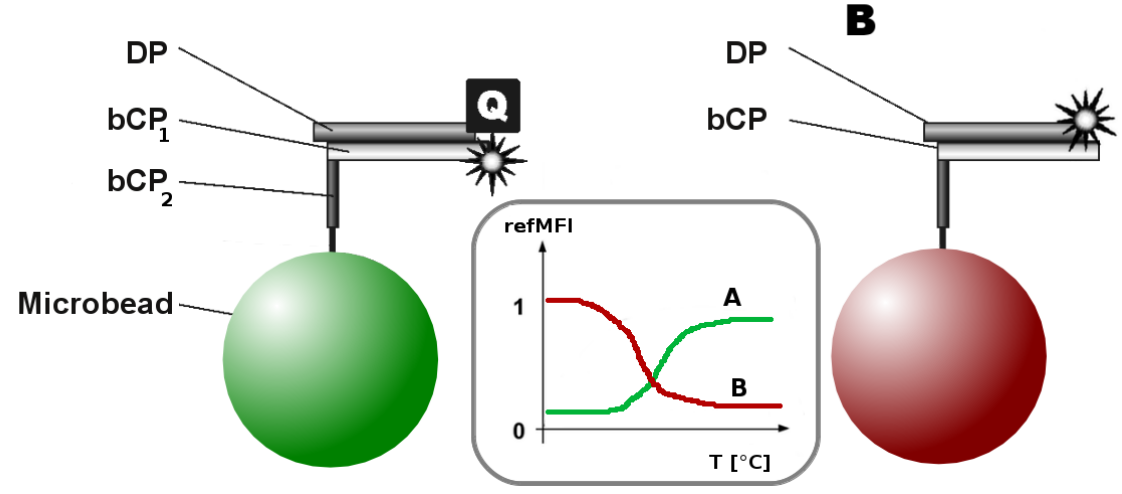

Figure 2: Principles of microbead direct hybridization probe systems. A) The FRET assay uses microbead bound capture probe $(b C P)$ with the fluorophore- $\left(^{*}\right)$ and quencher-labeled $(\mathbf{Q})$ detection probes $(D P)$. $\mathrm{A} b C P$ consists of different regions (e. g., $b C P_{1}, b C P_{2}$ ) ready to hybridize with a complementary $D P . \mathrm{B}$ ) The standard assay uses non-labeled $b C P$ s which hybridize with fluorophore-labeled DPs. Inset) Both probe systems differ in the curve shape resulting from the melting process.

\footnotetext{
${ }^{4}$ These include autoimmune cell pattern recognition, microbead-based assay for DNA and proteins. For details see Willitzki et al. (2012) and Rödiger et al. (2012b).

${ }^{5}$ The heating/cooling-unit (HCU) is based on peltier elements and has a performance similar to conventional thermal cyclers (e.g., iQ5 (Bio-Rad Laboratories)). For details see Rödiger et al. (2012b).
} 

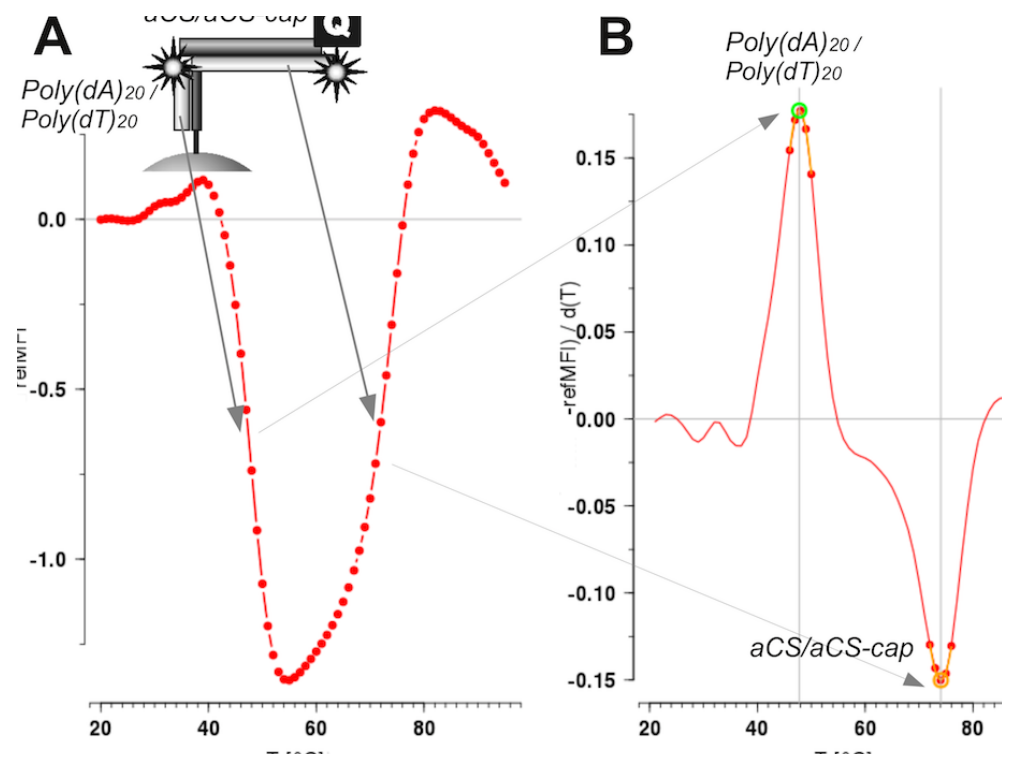

Figure 3: Melting curve and melting peaks. The data were obtained from a MCA experiment on microbead surfaces. Top left inset: Two DNA detection probes $\left(\operatorname{Poly}(d A)_{20}, a C S\right)$, were hybridized to a complementary microbead bound capture probe. A) While applying a temperature gradient $\left(20^{\circ} \mathrm{C}\right.$ to $95^{\circ} \mathrm{C}, 1{ }^{\circ} \mathrm{C}$ per step) the change of the refMFI is monitored. B) This probe system exhibits two melting peaks. Analysis showed the first (positive) peak for Poly $(d A)_{20}\left(\sim 48^{\circ} \mathrm{C}\right)$ followed by a second (negative) peak of $a C S\left(\sim 74^{\circ} \mathrm{C}\right)$.

Particularly, short 3' or 5' quencher/fluorophore-labeled probes were used ${ }^{6}$. The capture probes $(b C P)$ are complementary to detection probe $(D P)$ in solution (Figure 2 and inset Figure 13). Signals are mediated by temperature dependent dehybridization events between $b C P$ s and $D P$ s. All probe systems described here and our related works are characterized by one or two significant melting peaks at different temperatures and/or different signs (Figure 3). Due to different fluorophore / quencher combinations and probe systems (e. g., direct hybridization, dual-hybridization probes) the sign of a Tm peak value was designed to be either positive or negative (see Figure 3B). Transferred to applications in screening processes all subsequent analysis would be reduced to the identification of distinct $T m s$ and their intensity of the corresponding microbead populations.

\section{Why use R for Melting Curve Analysis?}

In biomedical research many scientists rely on closed source software which gives only little control over the computation and data presentation. Most importantly reproduction of data and calculations from other research is limited or impossible. Just recently this was discussed in the two journals Nature and Science (Ince et al., 2012; Morin et al., 2012). Closed software tied to limited tasks, hinders the import of custom data and gives no control over or insight into the source code and therefore is not ideal for research. Basically any open computing language or tool can be used to overcome these issues. But $\mathrm{R}$ fulfills all requirements mentioned above. It is in an open system with numerous utilities for data import, processing, sophisticated statistical analysis and presentation. Many $\mathrm{R}$ packages are peer-reviewed and undergo an intensive testing. Most importantly $\mathrm{R}$ is open source and therefore methods or results can be reproduced independently.

One implementation for MCA with $\mathrm{R}$ is available from the excellent qpcR package by Ritz and Spiess (2008). The meltcurve() function provides a sophisticated method to process melting curve data from qPCR experiments in solution. It uses automatic optimization procedures to smooth and fit curves. This function is ideal for the identification of multiple Tms, the calculation of the peak area and high resolution data.

${ }^{6}$ The DNA probe sequences, microbeads, probe immobilization process and experimental set up were described in Rödiger et al. (2011, 2012b). The fluorescence dye Atto 647N and the quencher BHQ2 were used generally. Intercalating dyes were not used since they are known to alter the melting process (Gudnason et al., 2007). 


\section{Motivation for the MBmca package}

There is an ongoing interest to understand the melting behavior of nucleic acids on surfaces. Particularly the effects of the reaction environment, e. g., the microbead surface change due to functional groups or the density of $b C P$ has not been investigated intensively for multiplex microbead assays. During the development of the VideoScan platform a simple and lightweight method for automatic screening, quality control and automatic detection of a limited number of melting peaks was needed. This article describes the $\mathrm{MBmca}^{7}$ (Roediger, 2013) package which was developed as an approach for statistical MCA on microbeads. The ambition of the MBmca is not to compete with the qpcR or other $\mathrm{R}$ packages but rather to extend the scope of $\mathrm{R}$ for MCA on surfaces.

\section{Implementation of MCA in the MBmca package}

\section{Functions and data sets of MBmca}

The MBmca package includes the functions MFIerror (), mcaPeaks ()$^{8}$ and mcaSmoother () for data inspection and preprocessing and diffQ( ), diffQ2() for MCA. The data sets DualHyb, DMP and MultiMelt are raw fluorescence data measured with the VideoScan platform (Rödiger et al., 2012b) on microbead surfaces. The data are arranged as data. frames starting in the first column with the temperature $\left({ }^{\circ} \mathrm{C}\right)$ followed by the fluorescence values (refMFI). The package has a dependency to robustbase (Rousseeuw et al., 2013) and uses mainly standard R functions. Elementary steps, e. g., data import, are similar to other $\mathrm{R}$ functions and thus used as described elsewhere (Venables et al., 2013).

\section{Inspection of raw fluorescence data}

One of the fundamental strengths of the MCA on microbead surfaces is the achievable multiplex level. The MFIerror() function was developed for a fast multiple comparison of the temperature dependent variance of ref MFI. MFIerror() returns an object of the class data. frame with columns "Temperature", "Location" (Mean, Median), "Deviation" (Standard Deviation, Median Absolute Deviation) and "Coefficient of Variation".

- The argument errplot (default) sets MFIerror() to plot the results. In the default setting (CV = FALSE) the mean with the standard deviations is plotted. Using the argument rob = TRUE the median and the median absolute deviation (MAD) are plotted instead of the mean and standard deviation.

- If $\mathrm{CV}$ is true the coefficient of variation $(\mathrm{CV})$ is plotted. Setting the argument RSD = TRUE shows the relative standard deviation (RSD) in percent.

In an example the mean raw fluorescence from multiplex melting curves of twelve microbead populations was evaluated for the probes HPRT1 and MLC-2v (Multimelt data set). The probe system used corresponds to Figure 2A. Ideally the variance between the twelve microbead populations is low. MFIerror () takes the first column of MultiMelt as temperature value and columns 2 to 13 for the probes HPRT1 and columns 14 to 25 for $M L C-2 v$, respectively.

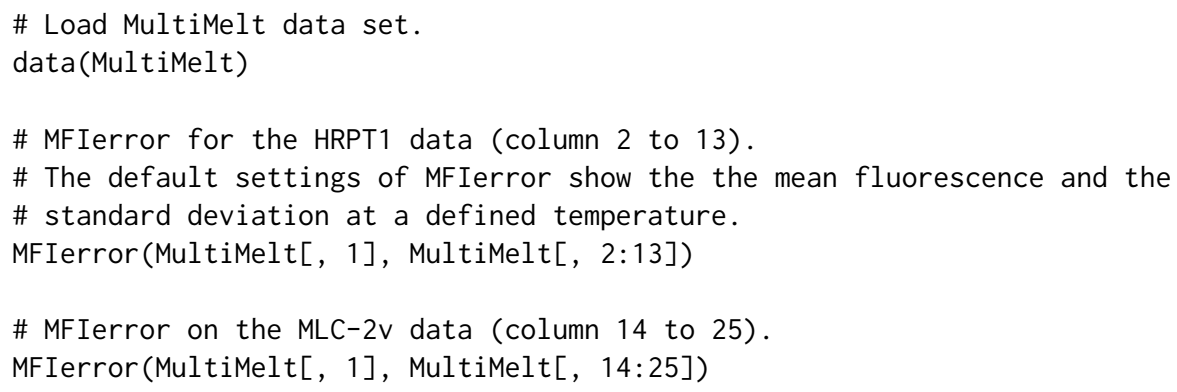

The corresponding plots are shown in Figure 4. The curves indicate that the different microbead populations show similar melting curve shapes but differ in height. At higher temperatures the values vary.

\footnotetext{
${ }^{7}$ MicroBead melting curve analysis.

8 mcaPeaks() is a function which can be used to estimate the number and location of the approximate local minima and maxima of melting curve data. This can be used to define a temperature range for MCA, melting curve quality control or peak height threshold definition (see Roediger 2013).
} 

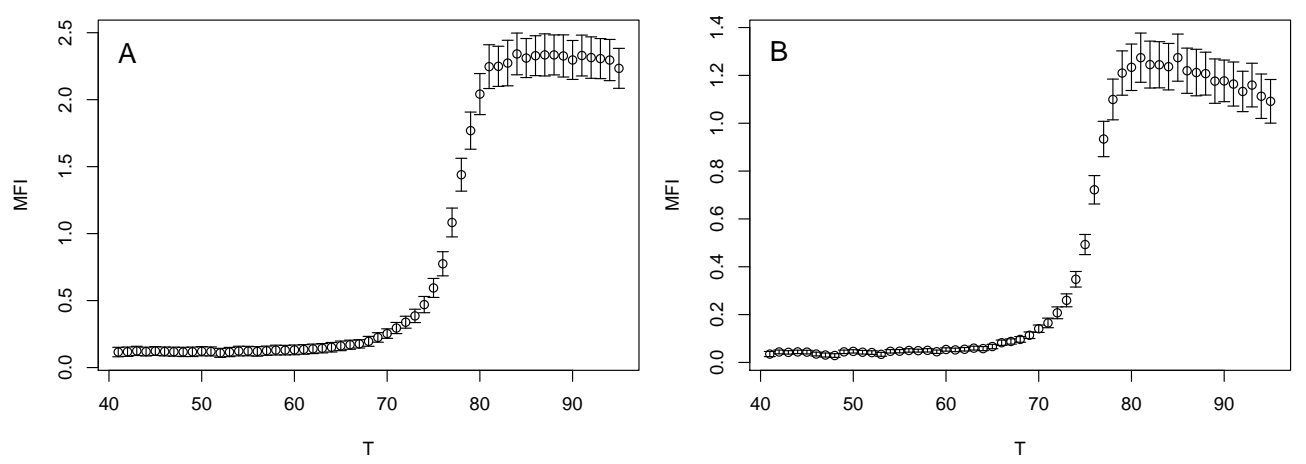

Figure 4: Inspection of the mean raw fluorescence from multiplex melting curves. MFIerror() with the argument $\mathrm{rob}=$ FALSE was used to compare the mean and the standard deviationof the temperature dependent fluorescence on twelve microbead populations for A) HPRT1 and B) MLC $-2 v$.
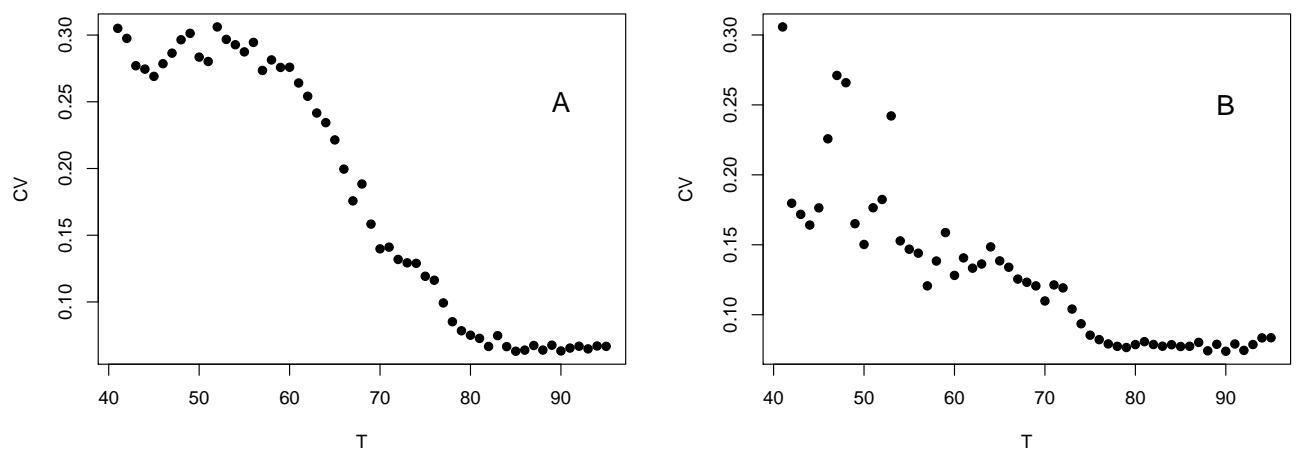

Figure 5: Comparison of the absolute coefficient of variation from multiplex melting curves. MFIerror() with argument CV = TRUE was used to plot the absolute coefficient of variation for twelve microbead populations with A) HPRT1 or B) $M L C-2 v$.

When MFIerror ( $)$ is used with the argument CV = TRUE the coefficient of variation is presented (Figure 5). In the example all $\mathrm{CV}$ values are low $(<0.3)$ and even decrease with increasing temperatures for both HPRT1 and MLC-2v.

We questioned how a single microbead population differs from the average of all microbead populations. The mean output of MFIerror(), with the argument errplot $=$ FALSE, was subtracted by the fluorescence of HPRT1 or MLC-2v. The results were assigned to HPRT1 mean and MLC2v. mean.

\# Load MultiMelt data set.

data(MultiMelt)

\# Use MFIerror to calculate the mean fluorescence for HRPT1 and MLC-2v over all

\# twelve microbead populations.

HPRT1.mean <- MFIerror(MultiMelt[, 1], MultiMelt[, 2:13], errplot = FALSE)

MLC2v.mean <- MFIerror(MultiMelt[, 1], MultiMelt[, 14:25], errplot = FALSE)

\# Draw figures on the graphics device in a $2 \times 6$ array

$\operatorname{par}(\mathrm{mfrow}=\mathrm{c}(2,6))$

\# Calculate the difference between the fluorescence of a single microbead population

\# and the average of all twelve microbead populations. Plot the results.

for (i in $1: 12$ ) \{

tmp.HPRT1 <- MultiMelt[, i + 1] - HPRT1.mean[, 2]

tmp.MLC2v <- MultiMelt[, i + 13]-MLC2v.mean[, 2]

$\operatorname{plot}($ MultiMelt $[, 1]$, tmp.HPRT1, main = paste ("Pop", i, sep = ": "), pch $=19$, ylim $\left.=c(-0.28,0.28), x l a b=" T^{\prime}, y l a b=" d e l t a "\right)$

abline (h $=0$, col $=$ "black")

abline $(v=65$, col = "blue")

\}

points(MultiMelt $[, 1]$, tmp.MLC2v, pch $=15, \operatorname{col}=2$ ) 

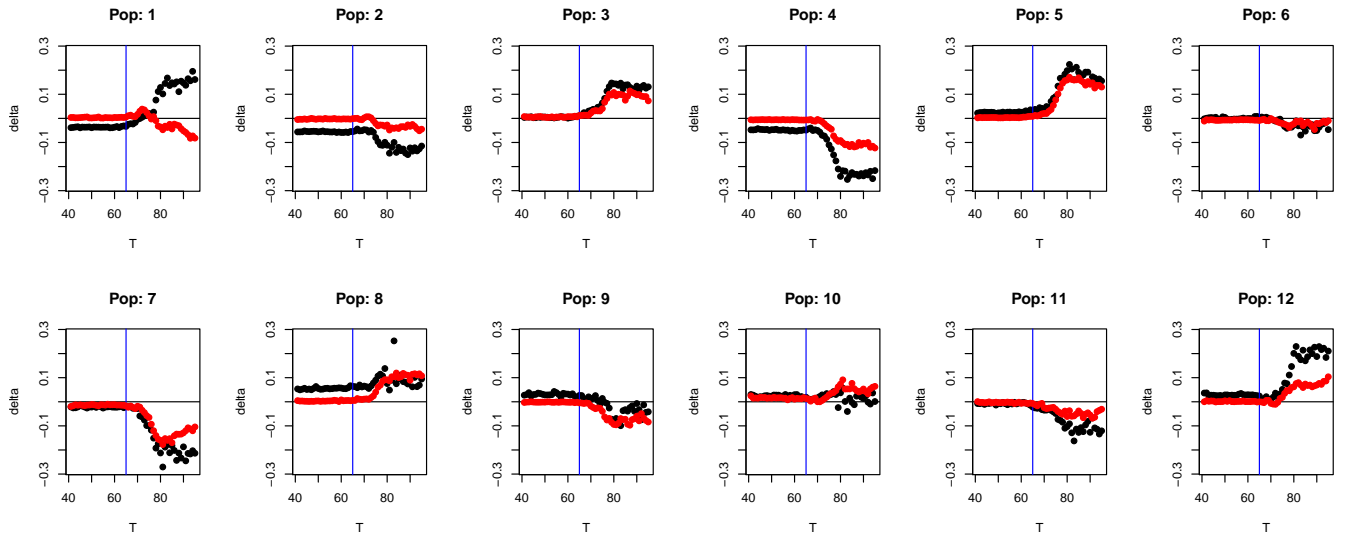

Figure 6: Difference plot of the fluorescence values. The temperature dependent mean fluorescence of twelve microbead populations, determined with MFIerror(), was subtracted from the fluorescence of single population ("Pop: "). • HPRT1, ・MLC-2v, — base line, — start of the melt process.

Figure 6 shows low differences (delta) at temperatures below $65^{\circ} \mathrm{C}$ due to near complete quenching. Above this temperature (start of the DNA probe strand dissociation) the differences to the mean fluorescence of all microbead populations grow. Apart from "Pop: 1" changes appear systematically which indicates that the $b C P / D P$ s have a similar melting behavior on all microbead populations.

\section{Preprocessing of raw fluorescence data}

The differentiation is the central step of the MCA. Accessible textbook information confirms that differentiation may result in the amplification of noise. To reduce the noise, $\mathrm{R}$ provides numerous possibilities to fit smooth functions and use filter functions. Such operations may alter the curve shape considerably and thus lead to artificial results. Smooth functions available in $\mathrm{R}$ include the moving average (filter(), stats), the LOWESS smoother (lowess(), stats) which applies locallyweighted polynomial regression, fitting of a local polynomial regression (loess(), stats), SavitskyGolay filter (sgolayfilt(), signal; The signal Developers, 2013), cubic splines (smooth.spline(), stats) or Friedman's SuperSmoother (supsmu(), stats). Although smoothed data might provide the impression of high quality data no guarantee for optimal results or that no peaks were artificially introduced is given. A good practice is to visualize the output combined with the original data. mcaSmoother() uses smooth.spline() and contains further helper function. mcaSmoother() should be used if the data may contain missing values, high noise or if the temperature resolution of the melting curve data is low $\left(\geq 0.5^{\circ} \mathrm{C} /\right.$ step $)$ in order to correct the problems automatically.

- Measurements from experimental systems may occasionally include missing values (NA). Function mcaSmoother ( ) uses approx ()$^{9}$ to fill up NAs under the assumption that all measurements were equidistant. The original data remain unchanged and only the NAs are substituted.

- mcaSmoother() calls smooth.spline() to smooth the curve. Different strengths can be set using the argument $d f$. fact (default 0.95). Internally it takes the degree of freedom value from the spline and multiplies it with a factor between 0.6 and 1.1. Values lower than 1 result in more strongly smoothed curves.

- If the argument bgadj is set TRUE, bg must be used to define a temperature range for a linear background correction $^{10}$. The linear trend is estimated by a robust linear regression using $\operatorname{lmrob}()$. In case criteria for a robust linear regression are violated $\operatorname{lm}()$ is used automatically.

- The argument Trange can be used to define a temperature range of the analysis.

- To scale the fluorescence a Min-Max normalization (Equation 6) between 0 and 1 can be used by setting the argument minmax to TRUE. This is useful if the fluorescence values between samples vary considerably, for example due to high background. An advantage of this normalization is the preservation of the relationships between the values. However, on surfaces normalization

\footnotetext{
${ }^{9}$ Besides approx() (stats) further functions are available from the zoo package (Zeileis and Grothendieck, 2005, e.g., na.approx(), na.spline()) and the delftfews package (Frasca, 2012, na.fill(), na. interpolate()). approx() was integrated since further dependencies are omitted and a linear interpolation is used.

${ }^{10}$ Some software packages include automatic linear or non-linear background correction which work not reliable in many cases. The examples in Figure 4 use identical probe systems (direct hybridization) but different $b C P / D P$ combinations. In theory the melting curve shape should be very similar. Particularly values at temperatures above $80^{\circ} \mathrm{C}$ vary strongly. Therefore only a simple linear background correction was implemented.
} 


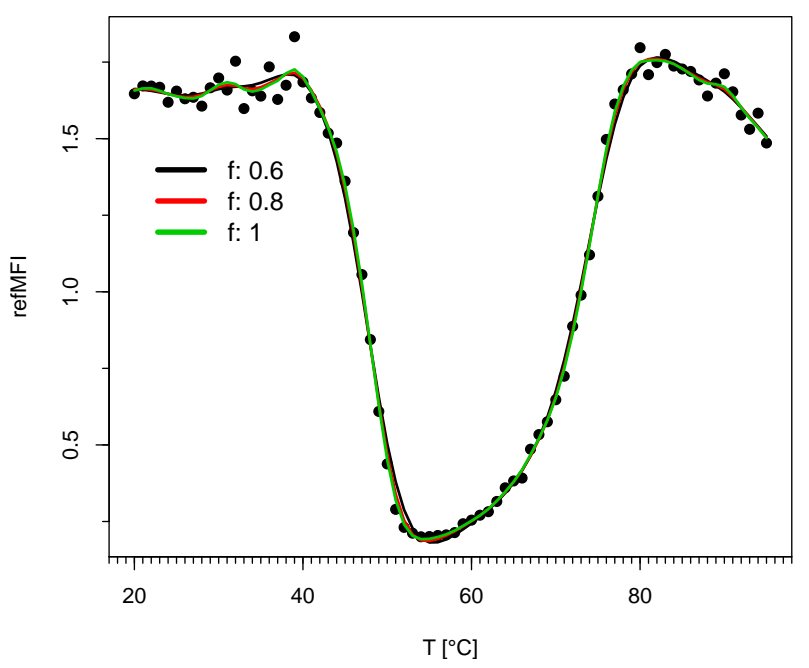

Figure 7: Raw fluorescence values $(\bullet)$ versus the temperature. Smoothed curves $(f: 0.6-, f: 0.8-$, $f: 1-)$ using mcaSmoother () with different strengths $(f)$ to smooth the curves.

should be used with caution because it might lead to the false impression that all microbeads carried equal quantities of $b C P$.

- The argument $\mathrm{n}$ uses the spline() function to increase the temperature resolution of the melting curve data by $n$-times the length of the input temperature (see mcaSmoother () examples in Roediger 2013).

$$
\text { refMFInorm }=\frac{r e f M F I-\min (r e f M F I)}{\max (r e f M F I)-\min (r e f M F I)}
$$

mcaSmoother () returns an object of the class "data.frame" with the columns " $x$ " (temperature) and " $y$ " (fluorescence values). These can be used to plot the preprocessed data. For example, three arbitrary chosen strengths to smooth the curves $(f: 0.6,0.8,1.0)$ were tested. Data from the DMP data set were used as follows:

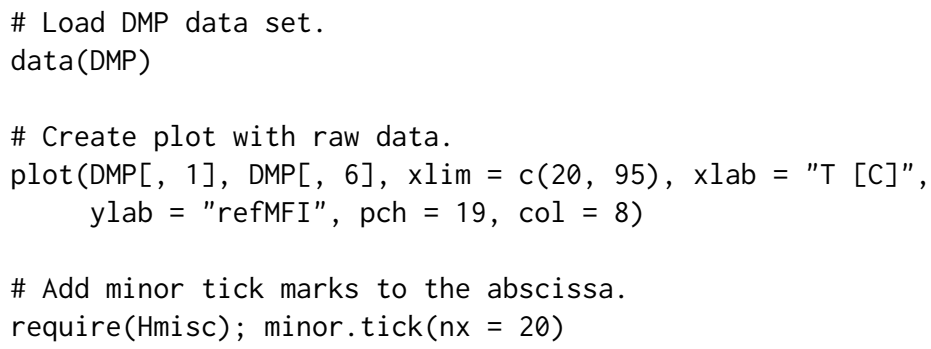

The function mcaSmoother ( $)$ is used in a loop to smooth the curve with user defined strengths $(f)$. Wrapped into lines() it draws ${ }^{11}$ the corresponding curves directly (Figure 7 ). In comparison to the original data a low filter strength $(f: 1)$ is sufficient.

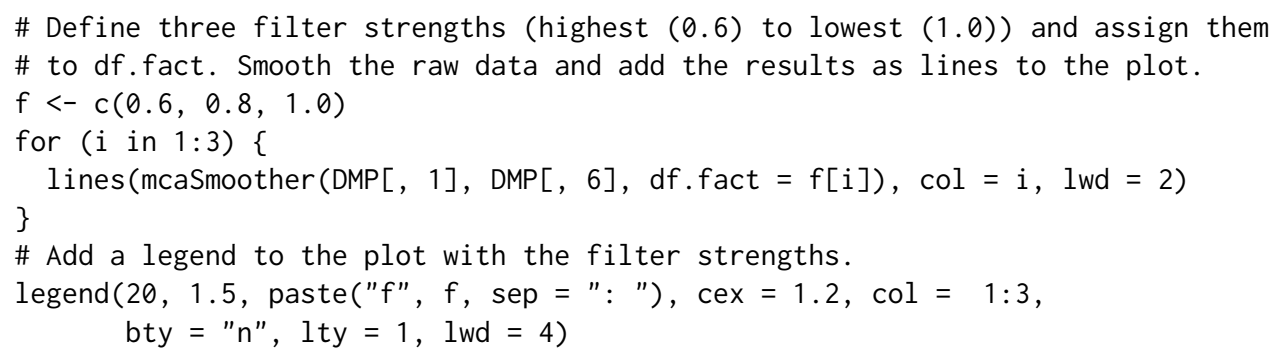

In the next example mcaSmoother() was used with different arguments to (i) smooth the data, (ii) remove the background and (iii) to perform a Min-Max normalization. Data for HPRT1 (Figure 8A) were taken from the MultiMelt data set. The plot of Figure $8 \mathrm{~B}$ implies that it is sustainable to smooth

\footnotetext{
${ }^{11}$ A fine grained abscissa was created with minor.tick() from the Hmisc package (Harrell Jr et al., 2013) to enhance the plot.
} 

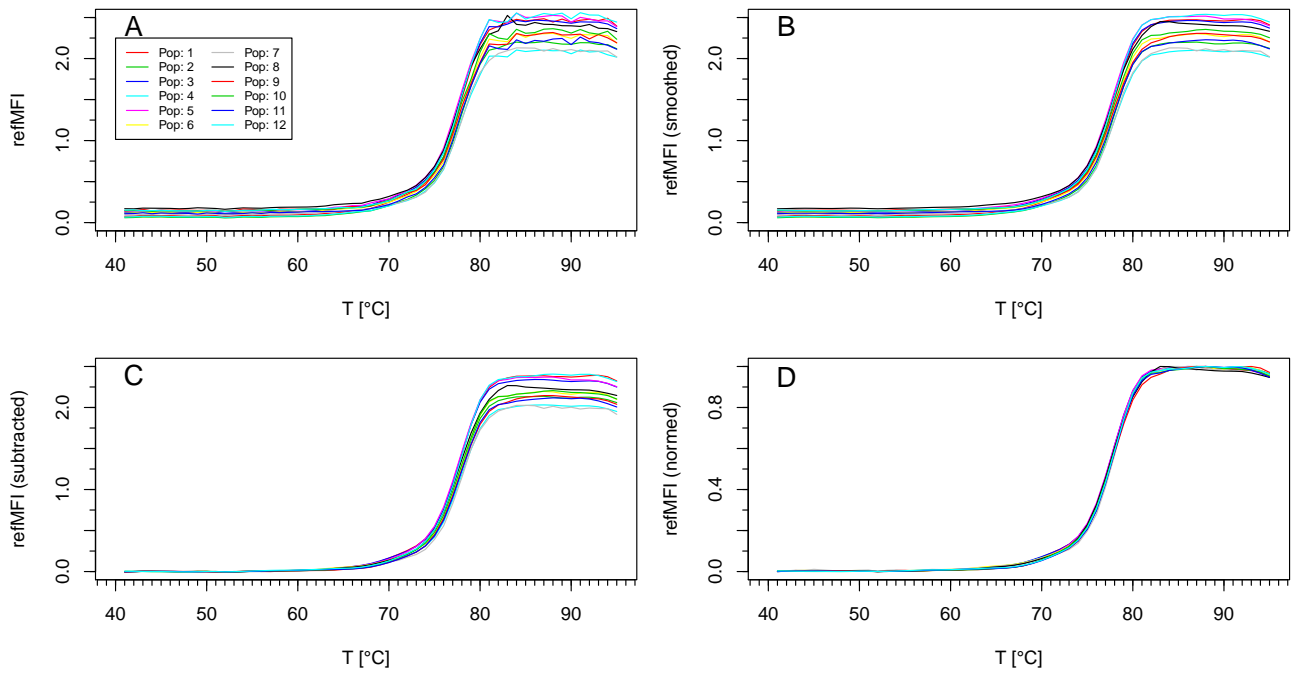

Figure 8: Preprocessing of HPRT1 with mcaSmoother (). A) Raw fluorescence data of melting curves from 12 microbead populations ("Pop: "). B) Smoothed curves with the default settings ( $f: 0.95)$. C) The smoothed curves after background reduction $\left(b g=c(41,61)\right.$, i.e., $41^{\circ} \mathrm{C}$ to $\left.61^{\circ} \mathrm{C}\right)$ and linear trend correction. D) Min-Max normalized curves.

the curve. Not immediately obvious, the twelve microbead populations have different final signal intensities. This is due to the different quantities of surface bound $b C P \mathrm{~s}$ (not shown). However, this is easily visualized after a background correction (Figure 8C). All curves appear similar in shape after the Min-Max normalization. This indicates that there are no substantial differences between the microbead populations which obfuscates further MCAs (Figure 8D).

\section{Calculation of the melting peaks}

The functions $\operatorname{diffQ(})$ and diffQ2() are used to calculate $T m, T m_{1}^{D 2}$ and $T m_{2}^{D 2}$ (Figure 1) and to perform simple graphical operations (e. g., show derivatives). Basically, both functions do not require smoothed data ${ }^{12}$ for the MCA. However, it is recommended to use mcaSmoother () as a starter function to preprocess (e.g., moderate smoothing, missing value removal, type check) the data automatically. First the approximate $T m, T m_{1}^{D 2}$ and $T m_{2}^{D 2}$ are determined as the $\min ()$ and/or $\max ()$ from the derivatives $^{13}$ according to Equation 1, 3 and 4 . This approximate peak value is the starting-point for an accurate calculation. The function takes a defined number $n$ (maximum 8 ) of the left and the right neighbor values and fits a quadratic polynomial ${ }^{14}$. The quadratic regression $\operatorname{lm}(Y \sim X$ $I\left(X^{\wedge} 2\right)$ ) of the $X$ (temperature) against the $Y$ (fluorescence) range gives the coefficients. The optimal quadratic polynomial is chosen based on the highest adjusted $R$-squared value $\left(R_{a d j .}^{2}\right)$. In the example of Figure 10 two left and right neighbors were required. The coefficients are used to calculate the root of the quadratic polynomial and thus to determine $T m, T m_{1}^{D 2}$ and $T m_{2}^{D 2}$. An estimate of a $T m$ does not neccesarily reflect a valid result. Therefore, several routines were implemented in diffQ() and diffQ2() which try to catch cases where an experiment went wrong. This includes a test if the data originate from noise, a test which analyses the difference between the approximate $\mathrm{Tm}$ and the calculated $T m$, and a tests for the goodness of fit value $\left(R_{a d j .}^{2}\right.$, Normalized-Root-Mean-Squared-Error (NRMSE)) of the quadratic polynomial. The functions will give a warning message and point to the potential error ${ }^{15}$. A good practice is to visualize the output and to control the peak heights (Example in Section "Multiplex analysis of dual melting peaks"). A bimodal probe system (compare Figure $3 \mathrm{~B}$ ) requires the separation of the analysis. The minimum and maximum of the approximate first derivative have to be determined independently. Although diffQ() is a major function it has only a simple plot function. By setting the argument plot = TRUE plots for single melting curves can be investigated (Figure 9). diffQ() accepts further following arguments:

\footnotetext{
${ }^{12}$ The paramter $\mathrm{rsm}$ is avilable in both functions to double the temperature resolution. This may also reduce noise.

${ }^{13}$ Besides the algorithm used in $\operatorname{diffQ()}$ there are further ways to calculate the approximate derivative in $\mathrm{R}$ such as $\operatorname{diff()}$ (base) or functions from the fda package (Ramsay et al., 2013).

${ }^{14}$ Quadratic polynomials are a good compromise because they are easy to implement, do not tend to swing and fit non-linear progresses sufficiently flexible.

${ }^{15}$ See example for diffQ() in Roediger (2013).
} 

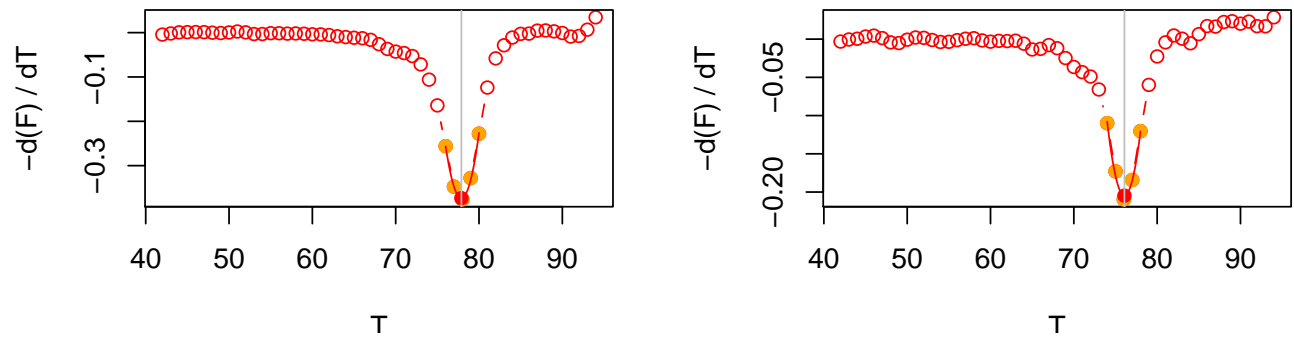

Figure 9: By default diffQ() shows no melting peak plot. diffQ(), with the argument plot = TRUE, can be used to show the melting peak (red dot) and fitted region from the quadratic polynomial (line) of a single melting curve. In this example HPRT1 (left) and MLC-2v (right) from MultiMelt are used.

- fct accepts min or max as argument and is used to define whether to find a local minimum ("negative peak") or local maximum ("positive peak").

- fws defines the number $(n)$ of left and right neighbors to use for the calculation of the quadratic polynomial.

- plot defines if a single plot of the melting peak should be created. If FALSE (default) no plot is created.

- negderiv is used to change the sign of the derivatives. If TRUE (default) then the first negative derivative is calculated. This argument was implemented to compare different quencher / fluorophore combinations (compare Figure 2).

- Functions for a graphical output include peak to show the peak values and deriv to show the first derivative with the color assigned to col. derivlimits and derivlimitsline show the number of neighbors $(n)$ or the region used to calculate the Tm. vertiline draws a vertical line at the Tms (Figure 10).

\# Load MultiMelt data set.

$\operatorname{data(MultiMelt)}$

\# Draw figures on the graphics device in two columns.

$\operatorname{par}(\operatorname{mfrow}=c(1,2))$

\# Use mcaSmoother to check and smooth the raw data for HRPT1 (2) and MLC-2v (14)

\# with the default setting.

\# Plot the first derivative of the two samples.

for (i in $c(2,14)$ ) \{

tmp <- mcaSmoother(MultiMelt[, 1], MultiMelt[, i $]$ )

diffQ(tmp, plot $=$ TRUE, vertiline $=$ TRUE)

\}

For sophisticated analysis and plots it is recommended to use diffQ() as part of the procedure. The arguments (e.g., peak, deriv) can be used when a plot already exists. diffQ2() calls instances of diffQ() to calculate $T m_{1}^{D 2}$ and $T m_{2}^{D 2}$. The arguments are similar to diffQ(). Both diffQ() and diffQ2() return objects of the class list. Accessing components of lists is done as described elsewhere (Venables et al., 2013; Roediger, 2013) either by name or by number.

\section{Practical applications}

\section{Multiplex analysis of single melting peaks}

diffQ2() was used to investigate effects of the surface capture probe density which is represented by the maximal refMFI value. The $T m, T m_{1}^{D 2}$ and $T m_{2}^{D 2}$ values were determined simultaneously on 12 microbead populations (12-plex) either for HPRT1 (compare Figure 8) or MLC-2v using data from MultiMelt. In the following HPRT1 was used as example. The corresponding matrix was called HPRT ${ }^{16}$.

\# Load MultiMelt data set. $\operatorname{data(MultiMelt)}$

\footnotetext{
${ }^{16}$ The script for $M L C-2 v$ is similar with the exception that the indices to access the elements of the data. frame need to be adapted accordingly.
} 

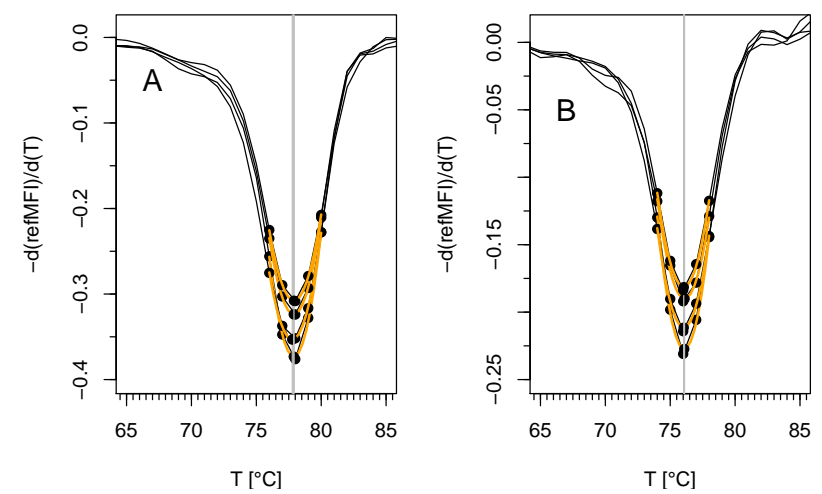

Figure 10: Output of $\operatorname{dif} f()$ and fitted region from the quadratic polynomial (orange line) for A) HPRT1 and B) MLC $-2 v$ of four randomly selected data from MultiMelt.

\begin{tabular}{lccc}
\hline$D P$ & $T m$ & $T m_{1}^{D 2}$ & $T m_{2}^{D 2}$ \\
\hline$M L C-2 v$ & $76.08 \pm 0.04$ & $73.99 \pm 0.11$ & $78.51 \pm 0.13$ \\
HPRT1 & $77.85 \pm 0.08$ & $75.39 \pm 0.20$ & $80.31 \pm 0.14$ \\
\hline
\end{tabular}

Table 1: Results of $T m, T m_{1}^{D 2}$ and $T m_{2}^{D 2}$ quantification for $M L C-2 v$ and HPRT1.

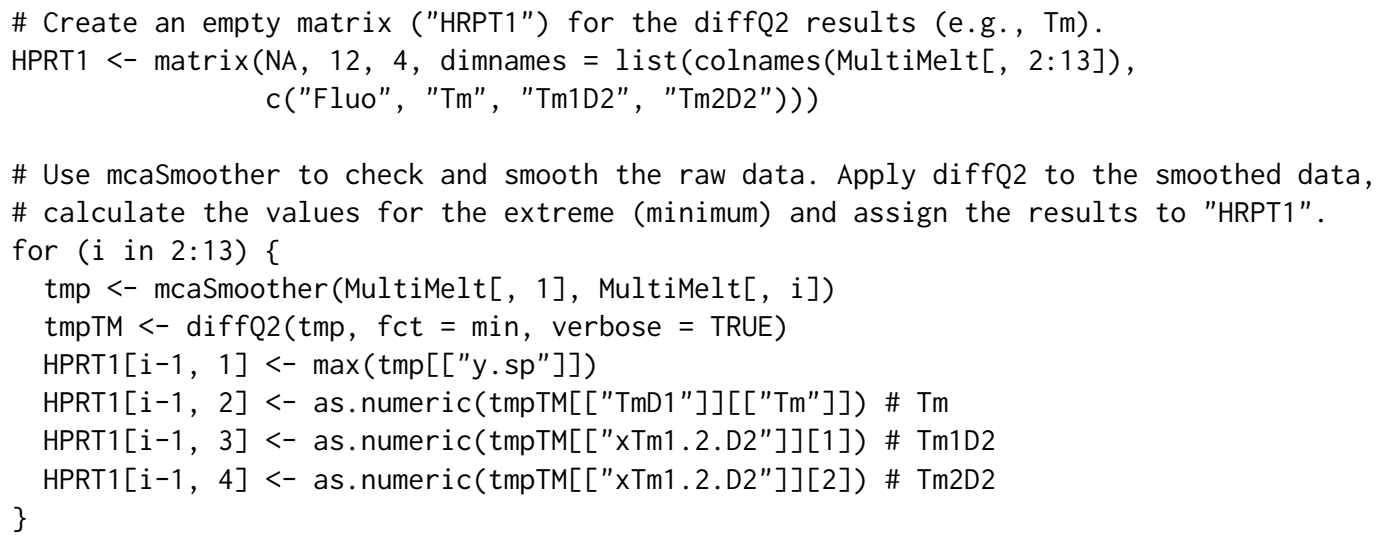

The surface capture density was determined by $\max \left(\operatorname{tmp}\left[\left[" \mathrm{y} . \mathrm{sp}^{\prime}\right]\right]\right)$. Subsequently the data from the matrices HPRT1 and MLC2v were plotted (Figure 11).

\# Plot the Tm, Tm1D2 and Tm2D2 form the matrix "HRPT1" versus the surface capture \# probe density ("Fluo").

plot(HPRT1 [, 1], HPRT1[, 2], xlab = "refMFI", ylab = "T [C]", main = "HPRT1", $\operatorname{xlim}=c(2.1,2.55), \operatorname{ylim}=c(72,82), \operatorname{pch}=19, \operatorname{col}=1: 12, \quad$ cex $=1.8)$

\# Add minor tick marks to the abscissa.

require(Hmisc); minor.tick $($ ny $=10)$

points(HPRT1 [, 1], HPRT1 [, 3], pch = 15)

points(HPRT1 [, 1], HPRT1[, 4], pch = 15)

\# Add trend lines $(\operatorname{lm}())$ for the peak values.

abline (lm(HPRT1[, 2] HPRT1[, 1])) \# Tm

abline (Im(HPRT1 $[, 3] \sim \operatorname{HPRT1}[, 1]))$ \# Tm1D2

abline $(\operatorname{lm}(\operatorname{HPRT} 1[, 4] \sim \operatorname{HPRT} 1[, 1]))$ \# Tm2D2

The melting temperature of $M L C-2 v$ was $76.08 \pm 0.04{ }^{\circ} \mathrm{C}$ and $77.85 \pm 0.08{ }^{\circ} \mathrm{C}$ for HPRT1 on all microbead populations (Table 1). This indicates that the surface capture probe density did not decrease or increase the $T m$ within the given range. $T m_{1}^{D 2}$ and $T m_{2}^{D 2}$ showed a symmetrical pattern with a low variance and therefore support that the start and end of the melting process are similar between the microbead populations. We suggest that the later peak values can be used as an additional means to describe the melting process in more detail. 
HPRT1

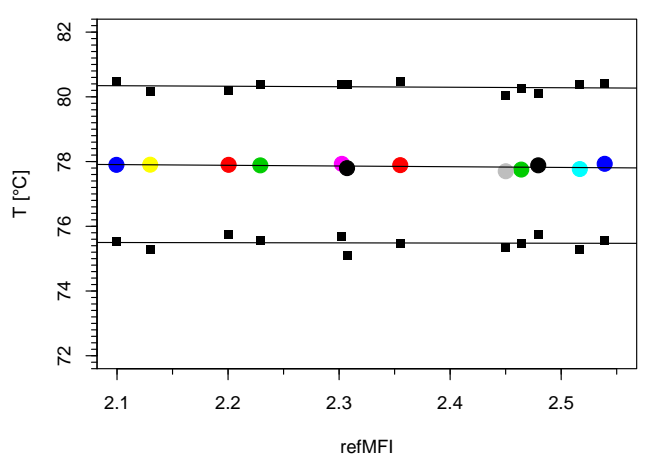

MLC-2v

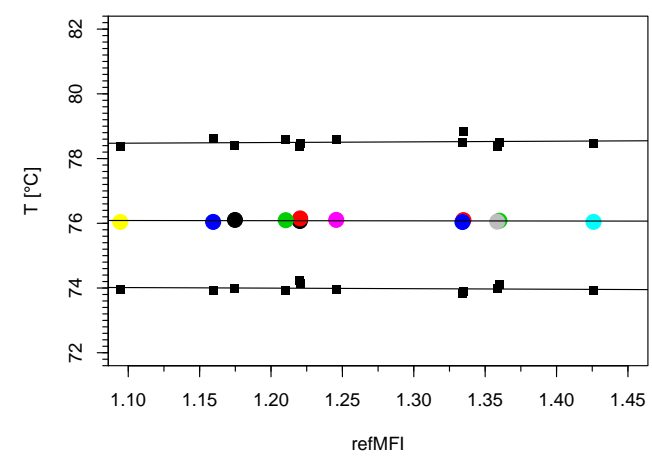

Figure 11: The peak values as function of capture probe density (sorted refMFI). Each colored dot represents the Tm and black squares the $T m_{1}^{D 2}$ and $T m_{2}^{D 2}$ of HPRT1 or MLC-2v on one microbead population.

\section{Multiplex analysis of dual melting peaks}

The $b C P$ s were hybridized with $D P$ s (compare Figure 3) in order to generate bimodal melting peak patterns ${ }^{17}$. Due to the base composition Poly $(d A)_{20} D P$ s were expected to melt at lower temperatures than the DPs $a C S$ and $M L C-2 v$. First the temperature and selected probes fluorescence values from the DMP data set were arranged in the data frame data. tmp and an empty plot was created.

\# Load DMP data set.

$\operatorname{data}(\mathrm{DMP})$

\# Use the temperature (column 1) and fluorescence (column 3, 5, 6), assign them to \# a temporary data frame and add the sample names.

data.tmp <- data.frame(DMP $[, 1], \operatorname{DMP}[, 3], \operatorname{DMP}[, 5], \operatorname{DMP}[, 6])$

names (data.tmp) <- c("T [C]", "Poly (dA)20 \& MLC-2v",

"Poly (dA)20 \& aCS", "Poly (dA)20")

\# Create a plot with the selected raw data.

$\operatorname{plot}(N A, N A, x \lim =c(20,95), y \lim =c(-0.6,0.6), x \operatorname{lab}=" T[C] "$,

$\mathrm{ylab}="-\mathrm{d}(\operatorname{refMFI}) / \mathrm{d}(\mathrm{T}) "$, main $=" "$, pch $=19$, col $=1: 12$, cex $=1.8$ )

\# Add minor tick marks to the abscissa.

require(Hmisc); minor.tick $(n x=10)$

Thereafter, the data were preprocessed with mcaSmoother () in a loop. The arguments bg $=c(20,35)$ and bgadj were used to adjust the background signal. This causes mcaSmoother () to use the subset of the data between $20^{\circ} \mathrm{C}$ and $35^{\circ} \mathrm{C}$ for the linear regression and background correction. To determine the $T m$ of the first probe (positive sign) and the second (negative sign) probe diffQ() was used with min and max for argument fct, respectively. In the loop the corresponding $T m$ values were assigned to the matrix RES and the melting curve is drawn. In addition to the lines the Tms were added (Figure 12).

\# Create an empty matrix ("RES") for the results of the peak values (Tm) and peak \# heights (F).

RES <- matrix(NA, 3, 4, dimnames = list (colnames $($ data.tmp[, 2:4]), $c(" F$ 1", "Tm 1", "F 2", "Tm 2")))

\# Use mcaSmoother to preprocess the raw data.

\# Use a background correction (20-35 degree Celsius).

\# Apply the smoothed data to diffQ, calculate the peak values for the extremes

\# (minimum and maximum) and assign the results to the matrix "RES".

\# Plot the smoothed data with the peak values and peak heights.

for (i in $c(1: 3)$ ) \{

tmp <- mcaSmoother (data.tmp $[, 1]$, data. $\operatorname{tmp}[, i+1]$, bgadj $=$ TRUE, $b g=c(20,35)$ )

lines (data.frame (diffQ (tmp, verbose $\left.=\operatorname{TRUE})\left[" x y^{\prime \prime}\right]\right)$, col = i)

$\operatorname{RES}[i, 1]<-\operatorname{round}(\operatorname{diffQ}(\mathrm{tmp}, \operatorname{fct}=\max )[[2]], 2) \quad$ \#fluoTm

$\operatorname{RES}[i, 2]<-\operatorname{round}(\operatorname{diffQ}(\mathrm{tmp}, \mathrm{fct}=\max )[[1]], 2) \quad$ \#Tm

${ }^{17}$ See Rödiger et al. (2012b) for further details of the probe system. 


\begin{tabular}{lll|lll}
\hline$D P / b C P$ & $F_{1}$ & $T m 1$ & $D P / b C P$ & $F_{2}$ & $T m 2$ \\
\hline Poly $(d A)_{20}$ & 0.59 & 49.6 & $M L C-2 v$ & -0.57 & 74.7 \\
- & 0.02 & 91.7 & Poly $(d A)_{20}$ & -0.08 & 49.7 \\
Poly $(d A)_{20}$ & 0.20 & 47.9 & $a C S$ & -0.15 & 73.9 \\
\hline
\end{tabular}

Table 2: Tm and peak values $\left(F_{1}, F_{2}\right)$ of the derivatives of bimodal melting curves. The detection probe and microbead bound capture probe pairs $(D P / b C P)$ of Poly $(d A)_{20} / \operatorname{Poly}(d T)_{20}$, $M L C-2 v / M L C-2 v-c a p$ and $a C S / a C S-c a p$ were analyzed simultaneously.

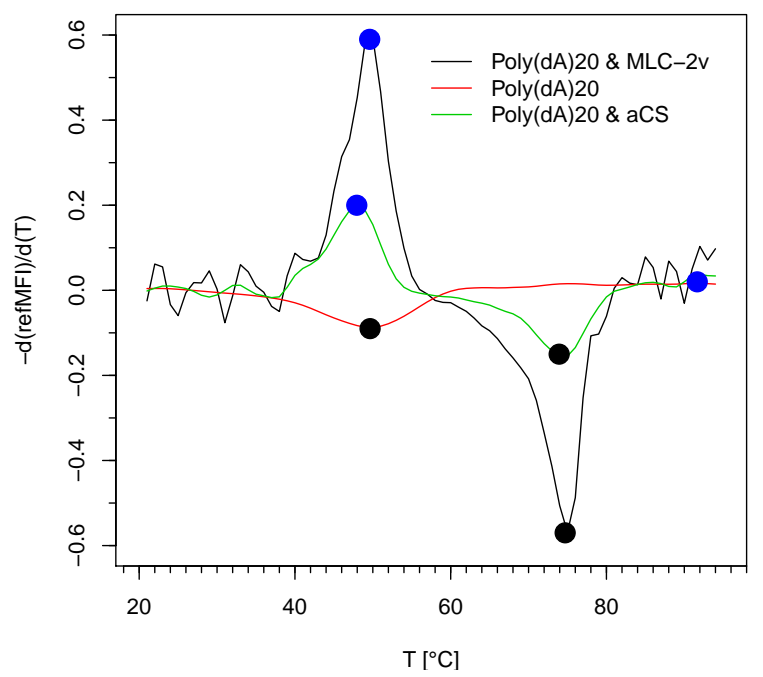

Figure 12: Melting peak pattern of $\operatorname{Poly}(d A)_{20}, M L C-2 v$ and $a C S$. Note: The sign of Poly $(d A)_{20}(-)$ is negative because the FRET assays was used (see Figure 2 and Rödiger et al. 2012b for details).

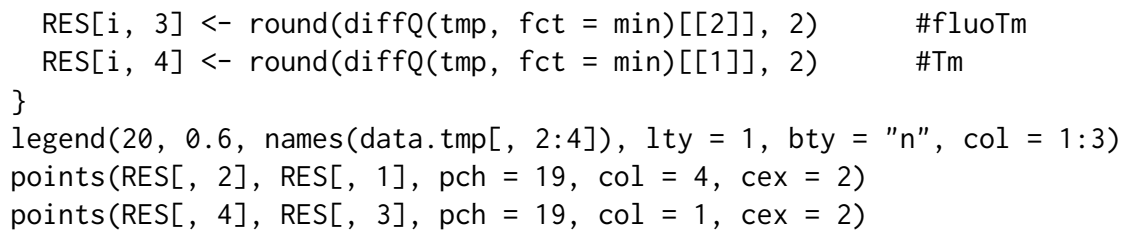

Figure 12 shows that $\operatorname{Poly}(d A)_{20}$ melts as single sharp peak at circa $48^{\circ} \mathrm{C}$. The detection probes $a C S$ and $M L C-2 v$ had a single peak at a higher $T m$ of circa $74^{\circ} \mathrm{C}$. Both, the number and the separation of the peaks were consistent to the estimated theoretical temperatures ${ }^{18}$ (not shown). The results of the matrix RES are shown in Table 2. The Tm of Poly $(d A)_{20}$ alone and Poly $(d A)_{20}$ combined with another detection probe differed slightly. This is presumably due to different capture immobilization strategies and /or $b C P / D P$ interactions (unpublished data).

\section{Multiplex SNP detection}

SNPs are important diagnostic markers for example in cardiac diseases (Villard et al., 2005; Muthumala et al., 2008). SNPs alter thermodynamic properties of dsDNA and thus the $\mathrm{Tm}$. In proof-of-principle experiments melting curves were obtained from sequences of human VIM with a single base exchange ${ }^{19}$. We used the previously reported (Rödiger et al., 2012b) dual-hybridization probe on microbeads to analyze the Tm shift (inset Figure 13).

The temperature resolution was $0.5^{\circ} \mathrm{C}$ per step. The DualHyb data were preprocessed with mcaSmoother () in a loop. The Tm and intensity ( fluoTm) were stored in the matrix RES.

\# Load DualHyb data set.

data(DualHyb)

\# Create an empty matrix ("RES") for the results of the peak values (TmD1)

\footnotetext{
${ }^{18}$ The open source tool PerlPrimer by Marshall (2007) was used to estimate the theoretical temperatures.

${ }^{19}$ Cytosine (C) was exchanged to thymine (T) at position 41 of a $60 \mathrm{bp}$ VIM oligonucleotide (see inset of Figure 13).
} 


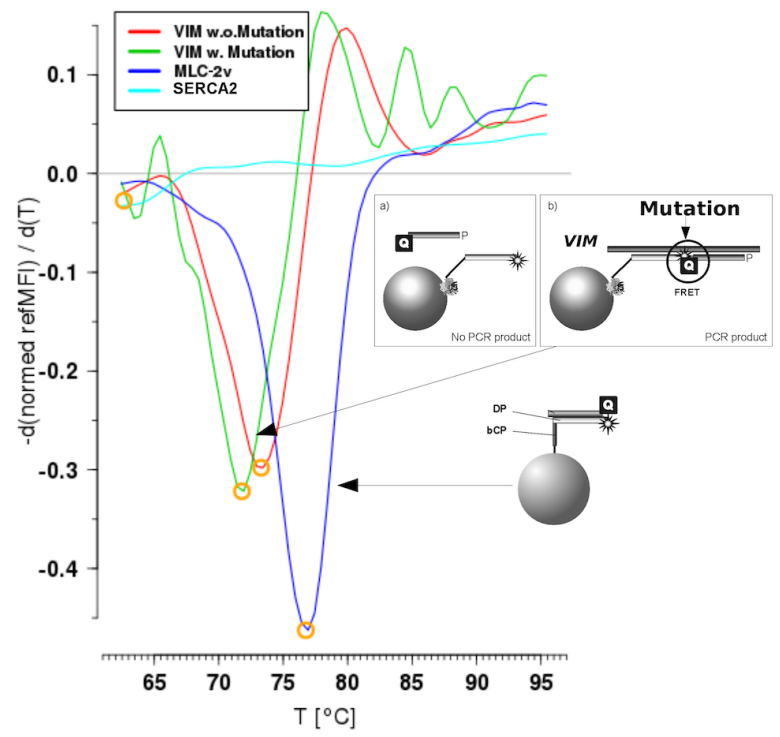

Figure 13: Proof-of-principle SNP detection with dual-hybridization assays on microbead surfaces. The melting temperature of the artificial mutated VIM $(-)(\mathrm{C} \rightarrow \mathrm{T})$ is lower compared to the native $V I M(-)$. MLC-2v (-) was used as reference for inter-assay variance. In the negative control SERCA2 (-) no significant Tm was detected.

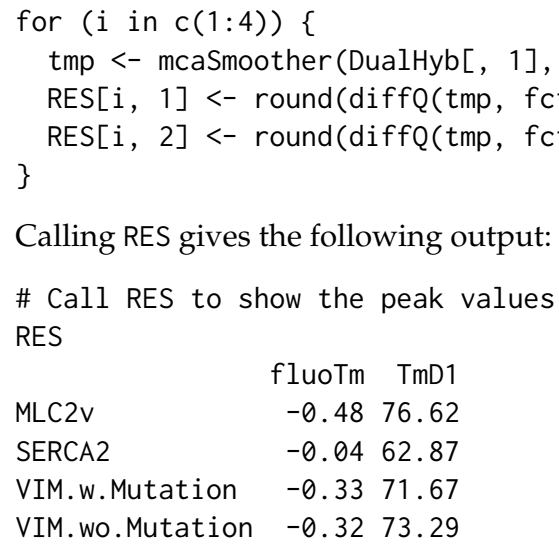

Calling RES gives the following output:

RES

$\begin{array}{lrr} & \text { fluoTm } & \text { TmD1 } \\ \text { MLC2v } & -0.48 & 76.62 \\ \text { SERCA2 } & -0.04 & 62.87 \\ \text { VIM.w.Mutation } & -0.33 & 71.67 \\ \text { VIM.wo.Mutation } & -0.32 & 73.29\end{array}$

\# Call RES to show the peak values and peak heights.

The algorithm calculated for the muted VIM a Tm of $71.67^{\circ} \mathrm{C}$ which is $1.62{ }^{\circ} \mathrm{C}$ lower than the native $V I M$ (Figure 13). This is in agreement with expected behavior. The negative control (unspecific $b C P$ for $S E R C A 2)$ had a $T m$ of $62.87^{\circ} \mathrm{C}$. But looking at the intensity showed that SERCA2 is very low $(-0.04)$. For screening purposes a simple cut-off would exclude this sample. The reference $M L C-2 v$ had a Tm of $76.62{ }^{\circ} \mathrm{C}$. Thus the method can be applied to identify SNPs. High multiplex level was achieved by using different capture probe microbead combinations. In this example a low temperature resolution of $0.5{ }^{\circ} \mathrm{C}$ per step was used. However, since lower heating rates are positively correlated with a higher resolution for SNP analysis generally higher resolutions are recommended. The temperature resolution of the raw data can be analyzed with $\operatorname{diffQ}(\ldots$, verbose $=\operatorname{TRUE})[[$ "temperature"] $]$.

\section{Summary and discussion}

Experimental hardware platforms often require the development of adapted software, in particular in cases where the hardware affects the signal (e. g., photo bleaching). R is an optimal tool for such scenarios. Within this article we proposed the MBmca package for MCA on microbead surfaces. The 
functions of the package were used for different detection-probe-systems, including direct hybridization of DNA dulexes or dual-hybridization probes for SNP detection. The capture-probe-systems produce unique melting profiles which allowed simple and rapid discrimination of different DNA sequences in one sample. The functions are useful to preprocess and inspect the data and to determine melting temperatures of immobilized DNA fragments. While used in this study for identification and quantification of biomolecules attached to microbeads it is applicable for MCA in solution too (not shown).

It was assumed that a quadratic polynomial at the approximate melting peaks can be used to calculate an accurate $T m$. One drawback is that the local quadratic polynomial use a predefined number $(n=1, \ldots, 8)$ of left and right approximate melting peaks neighbors. From experience this approach proved to be reliable for the temperature resolution of $0.5--1{ }^{\circ} \mathrm{C}$ per step as used in the present and the study of Rödiger et al. (2012b). Preliminary tests in solution using the LightCycler 2.0 (Roche) with high resolution $\left(\geq 0.1^{\circ} \mathrm{C}\right.$ per step) suggest that the method works too (not shown). This implementation is designed to meet the needs of a certain experimental setup and therefore may require evaluation prior to use in new applications. Besides $T m T m_{1}^{D 2}$ and $T m_{2}^{D 2}$ were proposed as additional values to describe early and late phases of the melting process on surfaces. Particularly, in investigations on the impact of the capture probe immobilization strategy or capture probe surface density these values might be useful. Methods to determine the area under the curve (AUC) were not taken into consideration due to the fact that photobleaching and quenching effects play an unknown role and are still an ongoing matter of debate in the literature. In practical terms it is recommended to implement functions from the MBmca package in an R GUI (see Valero-Mora and Ledesma 2012), e. g., RKWard ${ }^{20}$ (Rödiger et al., 2012a). GUIs provide more intuitive means to perform multiplex high-throughput analysis with visual feedback and enrichment with additional information like goodness of fit or peak heights.

\section{Acknowledgment}

Part of this work was funded by the BMBF InnoProfile-Projekt 03 IP 611. Grateful thanks belong to the authors of the cited R packages, the R community and the RKWard developers.

\section{Bibliography}

M. Frasca. delftfews: delftfews $R$ Extensions, 2012. URL http://R-Forge.R-project.org/projects/ delftfews/. R package version 0.3-163/r164. [p42]

M. B. Gašparič, T. Tengs, J. L. L. Paz, A. Holst-Jensen, M. Pla, T. Esteve, J. Žel, and K. Gruden. Comparison of nine different real-time PCR chemistries for qualitative and quantitative applications in GMO detection. Analytical and Bioanalytical Chemistry, 396(6):2023-2029, 2010. URL http: //www. ncbi.nlm.nih.gov/pubmed/20087729. [p37]

H. Gudnason, M. Dufva, D. Bang, and A. Wolff. Comparison of multiple DNA dyes for real-time PCR: Effects of dye concentration and sequence composition on DNA amplification and melting temperature. Nucleic Acids Research, 35(19):1-8, 2007. URL http://www. ncbi.nlm.nih.gov/pmc/ articles/PMC2095797/. [p39]

C. N. Gundry, J. G. Vandersteen, G. H. Reed, R. J. Pryor, J. Chen, and C. T. Wittwer. Amplicon melting analysis with labeled primers: A closed-tube method for differentiating homozygotes and heterozygotes. Clinical Chemistry, 49(3):396-406, 2003. URL http://www. ncbi.nlm.nih.gov/ pubmed/12600951. [p37]

F. E. Harrell Jr, C. Dupont, and et al. Hmisc: Harrell Miscellaneous, 2013. URL http://CRAN.Rproject. org/package=Hmisc. R package version 3.12-2. [p43]

D. C. Ince, L. Hatton, and J. Graham-Cumming. The case for open computer programs. Nature, 482 (7386):458-488, 2012. URL http://view.ncbi.nlm.nih.gov/pubmed/22358837. [p39]

O. Marshall. Graphical design of primers with PerlPrimer. Methods Molecular Biology, 402:403-414, 2007. URL http://www.ncbi.nlm.nih.gov/pubmed/17951808. [p48]

A. Morin, J. Urban, P. D. Adams, I. Foster, A. Sali, D. Baker, and P. Sliz. Shining light into black boxes. Science, 336(6078):159-160, 2012. URL http: //www. sciencemag. org/content/336/6078/159. [p39]

\footnotetext{
${ }^{20}$ Core structures of the MBmca were implemented in the RKWard melt plug-in (Rödiger et al., 2012a).
} 
A. Muthumala, F. Drenos, P. M. Elliott, and S. E. Humphries. Role of beta adrenergic receptor polymorphisms in heart failure: Systematic review and meta-analysis. European Journal of Heart Failure, 10(1):3-13, 2008. URL http://www. ncbi.nlm.nih.gov/pubmed/18158268. [p48]

J. O. Ramsay, H. Wickham, S. Graves, and G. Hooker. fda: Functional Data Analysis, 2013. URL http://CRAN. R-project. org/package=fda. R package version 2.4.0. [p44]

K. M. Ririe, R. P. Rasmussen, and C. T. Wittwer. Product differentiation by analysis of DNA melting curves during the polymerase chain reaction. Analytical Biochemistry, 245(9):154-160, 1997. URL http://www.ncbi.nlm.nih.gov/pubmed/9056205. [p37]

C. Ritz and A.-N. Spiess. qpcR: An R package for sigmoidal model selection in quantitative realtime polymerase chain reaction analysis. Bioinformatics, 24(13):1549-1551, 2008. URL http:// bioinformatics.oxfordjournals.org/content/24/13/1549.abstract. [p39]

S. Rödiger, M. Ruhland, C. Schmidt, C. Schröder, K. Grossmann, A. Böhm, J. Nitschke, I. Berger, I. Schimke, and P. Schierack. Fluorescence dye adsorption assay to quantify carboxyl groups on the surface of poly(methyl methacrylate) microbeads. Analytical Chemistry, 83(9):3379-3385, 2011. URL www.ncbi.nlm.nih.gov/pubmed/21413805. [p39]

S. Rödiger, T. Friedrichsmeier, P. Kapat, and M. Michalke. RKWard: A comprehensive graphical user interface and integrated development environment for statistical analysis with R. Journal of Statistical Software, 49(9):1-34, 2012a. URL http: //www. jstatsoft.org/v49/i09. [p37, 50]

S. Rödiger, P. Schierack, A. Böhm, J. Nitschke, I. Berger, U. Frömmel, C. Schmidt, M. Ruhland, I. Schimke, D. Roggenbuck, W. Lehmann, and C. Schröder. A highly versatile microscope imaging technology platform for the multiplex real-time detection of biomolecules and autoimmune antibodies. In Molecular Diagnostics, volume 133 of Advances in Biochemical Bioengineering/Biotechnology, pages 35-74. 2012b. doi: 10.1007/10_2011_132. [p37, 38, 39, 40, 47, 48, 50]

S. Roediger. MBmca: Nucleic Acid Melting Curve Analysis on Microbead Surfaces with R, 2013. URL http://CRAN. R-project.org/package=MBmca. R package version 0.0.2-1. [p40, 43, 44, 45]

P. Rousseeuw, C. Croux, V. Todorov, A. Ruckstuhl, M. Salibian-Barrera, T. Verbeke, M. Koller, and M. Maechler. robustbase: Basic Robust Statistics, 2013. URL http://CRAN. R-project. org/package= robustbase. R package version 0.9-10. [p40]

M. M. Sekar, W. Bloch, and P. M. St John. Comparative study of sequence-dependent hybridization kinetics in solution and on microspheres. Nucleic Acids Research,33(1):366-375, 2005. URL http: //www.ncbi.nlm.nih.gov/pmc/articles/PMC546151/. [p37]

The signal Developers. signal: Signal Processing, 2013. URL http://R-Forge.R-project.org/ projects/signal/. [p42]

P. M. Valero-Mora and R. Ledesma. Graphical user interfaces for R. Journal of Statistical Software, 49(1): 1-8, 2012. URL http://www. jstatsoft.org/v49/i01/paper. [p50]

W. N. Venables, D. M. Smith, and the R Core Team. An Introduction to R. R Foundation for Statistical Computing, Vienna, Austria, 2013. URL http://www. CRAN.R-project.org/doc/manuals/R-intro. pdf. [p40,45]

E. Villard, L. Duboscq-Bidot, P. Charron, A. Benaiche, V. Conraads, N. Sylvius, and M. Komajda. Mutation screening in dilated cardiomyopathy: Prominent role of the beta myosin heavy chain gene. European Heart Journal, 26(8):794-803, 2005. URL http://www. ncbi . nlm. ni h. gov/pubmed/15769782. [p48]

A. Willitzki, R. Hiemann, V. Peters, U. Sack, P. Schierack, S. Rödiger, U. Anderer, K. Conrad, D. P. Bogdanos, D. Reinhold, and D. Roggenbuck. New platform technology for comprehensive serological diagnostics of autoimmune diseases. Clinical $\mathcal{E}$ Developmental Immunology, 2012:1-8, 2012. URL https://www.ncbi.nlm.nih.gov/pubmed/23316252. [p38]

A. Zeileis and G. Grothendieck. zoo: S3 infrastructure for regular and irregular time series. Journal of Statistical Software, 14(6):1-27, 2005. URL http://www. jstatsoft. org/v14/i06. [p42]

L. Zhou, L. Wang, R. Palais, R. Pryor, and C. T. Wittwer. High-resolution DNA melting analysis for simultaneous mutation scanning and genotyping in solution. Clinical Chemistry, 51(10):1770-1777, 2005. URL http://www.ncbi.nlm.nih.gov/pubmed/16189378. [p37] 
Stefan Rödiger

Charité-Universitätsmedizin Berlin

and

Lausitz University of Applied Sciences

Faculty II

Germany

Stefan. Roediger@HS-Lausitz. de

Alexander Böhm

Lausitz University of Applied Sciences

Faculty I

Germany

Alexander. BoehmaHS-Lausitz.de

Ingolf Schimke

Charité-Universitätsmedizin Berlin

Germany

ingolf.schimke@charite.de 